\title{
Tracheomalacia and breath holding: a case report
}

\author{
H Griffiths, I Doull, R G Williams, C Marnane
}

\begin{abstract}
A child with a long standing history of cyanotic breath holding attacks presented with acute respiratory distress. Subsequent investigation established that her clinical condition was caused by tracheomalacia. We hypothesise that tracheomalacia might be an under recognised contributor to cyanotic breath holding attacks, the pathogenesis of which is poorly understood.

(Arch Dis Child 2000;83:340-341)
\end{abstract}

Keywords: tracheomalacia; breath holding; cyanotic

Breath holding attacks are common in infants and comprise the sequential events of apnoea, unconsciousness, and changes in postural tone triggered by adverse stimuli. Breath holding attacks usually begin before 2 years of age; $90 \%$ of sufferers are free of them by age $6 .{ }^{1}$ They are described as cyanotic or pallid, and further categorised as simple or complicated depending on whether seizures occur. Although it is thought that pallid (white) attacks are mediated through vagal mechanisms, the cause for cyanotic (blue) attacks is unclear. An autosomal dominant with reduced penetrance pattern of inheritance has been described, ${ }^{2}$ as has an association with iron deficiency. ${ }^{3}$

Tracheomalacia is caused by weakness of the tracheal wall caused by softening of the supporting cartilage, and hypotonia of the myoelastic elements. ${ }^{4}$ Characteristically the patient may have a barking cough, stridor, wheezing, and frequent respiratory infections. ${ }^{5}$ Primary tracheomalacia is thought to be caused by congenital immaturity of the tracheal cartilage, while in secondary tracheomalacia previously normal cartilage undergoes degeneration. ${ }^{6}$

\section{The case}

A 6 year old girl presented with a generalised convulsion following a prolonged breath holding attack. She had a history of severe breath holding attacks that often culminated in convulsions. Both her father and sister had had similar episodes until the age of 6 years.

In the six months prior to admission the patient had a history of recurrent chest infections which were attributed to her recently diagnosed ataxia telangectasia. Extubation proved difficult on two occasions because of the onset of stridor and respiratory acidosis. To facilitate weaning from the ventilator, a tracheostomy was performed. At operation the trachea was shown to be inflamed and oedematous, a finding consistent with prolonged and repeated intubation. The larynx was morphologically and functionally normal.
With a tracheostomy tube in situ the patient continued to have breath holding attacks. Flexible bronchoscopic examination was performed which revealed tracheobronchomalacia extending distal to the tip of the tracheostomy tube into the right main bronchus. An extra long tracheostomy tube was fitted to stent the malacic segment. Videofluoroscopy was performed and extrinsic compression of the trachea excluded.

The patient was discharged home with a hyperflex adjustable flange tracheostomy tube and speaking valve. Subsequently although the patient tried repeatedly to initiate breath holding attacks she was unable to do so.

\section{Discussion}

The pathophysiology of cyanotic breath holding attacks is unknown, but the natural history of the condition is that it improves with age, being uncommon after the age of 4.5 years. ${ }^{1}$ This patient had relatively severe breath holding attacks and a strong family history, supporting the diagnosis. The attacks continued in the presence of a standard tracheostomy tube; the fact that a longer tube corrected the condition leads us to believe that the tracheomalacia facilitated the breath holding attacks.

It is well established that during expiration, the calibre of the tracheal lumen decreases passively in response to the rise in intrathoracic pressure (this being more notable in children than adults). ${ }^{4}$ The active holding of the breath in cyanotic breath holding attacks also occurs during the expiratory phase of the respiratory cycle. ${ }^{1}$ This is in reality an involuntary valsava manoeuvre, with a consequent increase in the intrathoracic pressure, over and above that normally seen in expiration. The likely result for a patient with a malacic segment to their trachea with its increased compliance, is that its tendency to collapse and obstruct the airway is enhanced.

The natural history of primary tracheomalacia is similar to that of cyanotic breath holding attacks. They occur in a paediatric population of similar age, and have similar symptoms. Both regress spontaneously. We hypothesise that primary tracheomalacia might be an under recognised contributor to cyanotic breath holding attacks.

1 Evans OB. Breath holding spells. Pediatr Ann 1997;26:41014

2 Di Mario FJ Jr, Sarfarazi M. Family pedigree analysis of children with breath holding spells. F Pediatr 1997;130: 647-51

3 Hannan DW. Breath holding spells: waiting to inhale, waiting for systole, or waiting for iron therapy? 7 Pediatr 1997 ; 130:510-12

4 Baxter JD, Dunbar JS. Tracheomalacia. Ann Otol Rhinol Laryngol 1963;72:1013-23.

5 Benjamin B. Tracheomalacia in infants and children. Ann Otol Rhinol Laryngol 1984;93:438-42.

6 Thomas H, Cogbill, MD, Frederick A, et al. Primary tracheomalacia. Ann Thorac Surg 1983;35:538-41. 


\section{Commentary}

Breath holding attacks, affectionately known as kinking in Yorkshire, are said to occur in $0.1-5 \%$ of healthy children between the ages of 6 months and 6 years. The attacks usually start in the first year of life and are often provoked by upset, anger, or injury. Typically the child starts to cry and then becomes noiseless. Although the term "breath holding" implies they occur during inspiration, they usually occur during expiration and are reflex in nature. Following the period of breath holding, the child may start breathing again, lose consciousness, or in severe cases may progress to a seizure.

Tracheomalacia exists when the cartilaginous framework of the trachea is unable to maintain airway patency. The cartilage in the airway of infants is normally soft and therefore all infants have some degree of dynamic collapse during expiration, when the pressure outside the trachea exceeds the pressure inside. In tracheomalacia dynamic collapse leads to airway obstruction. Tracheomalacia is usually divided into congenital or acquired. The congenital variety may be associated with other developmental defects such as a vascular ring or tracheo-oesophageal fistula; it commonly involves the majority of the trachea and other major airways. The acquired form often follows neonatal lung disease and prolonged ventilation. Symptoms of tracheomalacia include wheeze, cough, stridor, dyspnoea, tachypnoea, cyanosis, and recurrent respiratory tract infections. Diagnosis can be difficult and is often based on an assessment of the airway at bronchoscopy. This is inevitably subjective and a general anaesthetic can mask dynamic airway collapse; alternatively obstruction of the airway with a bronchoscope can exacerbate the collapse.

The child described by the authors of this paper was apparently well until 5 and a half years old; she then had repeated respiratory tract infections over a six month period. It is not clear from the case report how old the child was when she started having breath holding episodes. The authors do not describe any of the typical symptoms of tracheomalacia prior to her episode of ventilation, other than possibly repeated chest infections. This child presumably had a period of prolonged ventilation following a convulsion. Extubation failed twice due to stridor and a respiratory acidosis; a tracheostomy was performed to facilitate weaning. The trachea was found to be inflamed and oedematous; this was thought to be due to repeated and prolonged intubation. Flexible bronchoscopy revealed tracheobronchomalacia. Fitting an extra long tracheostomy tube prevented breath holding attacks. The authors comment that the patient tried to initiate breath holding unsuccessfully.

The authors speculate that primary tracheomalcia may be an under recognised contributor to cyanotic breath holding attacks. I have been involved in the care of two cases of Larsen's syndrome, both with severe airway malacia and both with cyanotic breath holding episodes. These patients had significant chronic symptoms of tracheomalacia. In the case described in this paper there are no features of tracheomalacia prior to the episode of prolonged intubation. The trachea was clearly inflamed and therefore this could well have been acquired tracheomalacia. The breath holding episodes presumably began very early in life and predated any symptoms of tracheomalacia.

It is perhaps not surprising that with the airway splinted by a long tracheostomy tube it was not possible for the child to perform an involuntary valsava like manoeuvre and obstruct her airway. Equally it makes sense that a malacic trachea is more susceptible to collapse and it is therefore easier for these patients to induce an involuntary valsava manoeuvre. It has yet to be shown that children without chronic symptoms of tracheomalacia who have severe cyanotic breath holding episodes may have significant primary tracheomalacia.

Consultant Paediatrician

KEITH BROWNLEE Gledhow Wing

St fames's University Hospital

Leeds LS9 7TF, UK

email: keith.brownlee@gw.sjsuh.northy.nhs.uk 